\title{
Functional Outcomes of Billroth I Gastroduodenostomy Using Linear Staplers in Totally Laparoscopic Distal Gastrectomy
}

\author{
KEITA KATSURAHARA ${ }^{1 *}$, TOSHIYUKI KOSUGA ${ }^{1 *}$, TAKESHI KUBOTA ${ }^{1}$, ATSUSHI SHIOZAKI $^{1}$, \\ KAZUMA OKAMOTO ${ }^{1}$, KATSUTOSHI SHODA $^{1}$, HIROTAKA KONISHI ${ }^{1}$, HITOSHI FUJIWARA $^{1}$, \\ MICHIHIRO KUDOU ${ }^{1}$, TOMOHIRO ARITA ${ }^{1}$, RYO MORIMURA ${ }^{1}$, YASUTOSHI MURAYAMA ${ }^{1}$, \\ YOSHIAKI KURIU ${ }^{1}$, HISASHI IKOMA ${ }^{1}$, MASAYOSHI NAKANISHI ${ }^{1}$, SHUHEI KOMATSU ${ }^{1,2}$ and EIGO OTSUJI ${ }^{1}$ \\ ${ }^{1}$ Division of Digestive Surgery, Department of Surgery, Kyoto Prefectural University of Medicine, Kyoto, Japan; \\ ${ }^{2}$ Department of Gastroenterological Surgery, Japanese Red Cross Society Kyoto Daiichi Hospital, Kyoto, Japan
}

\begin{abstract}
Background/Aim: This study examined whether functional outcomes of linear-stapled Billroth I (LS-BI) in totally laparoscopic distal gastrectomy (TLDG) are comparable to those of circular-stapled Billroth I (CS-BI) in laparoscopy-assisted distal gastrectomy (LADG). Patients and Methods: This was a retrospective study of patients with gastric cancer undergoing TLDG with LS-BI $(n=50)$ or $L A D G$ with CS-BI $(n=50)$. Postoperative endoscopic findings of the remnant stomach and nutritional status were evaluated. Results: The occurrence of grade 2 or more severe remnant gastritis in the LS-BI group (46.0\%) was significantly higher than that in the CS-BI group (18.0\%) $(p=0.005)$, whereas there was no significant difference in the incidence of residual food and bile reflux between the two groups. Postoperative changes in body weight, and serum albumin and total protein levels were similar between the two groups. Conclusion: TLDG with LS-BI may be a good alternative to $L A D G$ with $C S-B I$ because of its comparable nutritional outcomes, but with a higher occurrence of remnant gastritis.
\end{abstract}

Since its first introduction in the early 1990s, laparoscopic distal gastrectomy (DG) has been increasingly used as a less invasive approach to surgery for gastric cancer in the middle

This article is freely accessible online.

*These Authors contributed equally to this study.

Correspondence to: Toshiyuki Kosuga, MD, Ph.D., Division of Digestive Surgery, Department of Surgery, Kyoto Prefectural University of Medicine, 465 Kajii-cho, Kamigyo-ku, Kyoto, 6028566, Japan. Tel: +81 752515527, Fax: +81 752515522, e-mail: toti-k@koto.kpu-m.ac.jp

Key Words: Gastric cancer, laparoscopic gastrectomy, Billroth I, gastritis, nutritional status. and lower third of the stomach $(1,2)$. The initial procedure was 'laparoscopy-assisted' distal gastrectomy (LADG) in which reconstruction was performed through a minilaparotomy, while use of 'totally laparoscopic' distal gastrectomy (TLDG) with intracorporeal anastomosis has gradually increased with advances in surgical techniques (3).

In LADG, Billroth I gastroduodenostomy (BI) is usually completed with a circular stapler (CS) in a similar fashion to that employed in open surgery (2). In contrast, BI in TLDG commonly employs only linear staplers (LS) because of their easier application through trocars and easier insertion into the duodenum. Several techniques for intracorporeal BI using LSs, such as delta-shaped anastomosis, the augmented rectangle technique and the book-binding technique, have been reported, with excellent short-term outcomes (4-6); however, few studies examined their long-term outcomes. Therefore, it remains unclear whether the postoperative functional outcomes of LS-BI are comparable to those of CS-BI. While the wider lumen of anastomosis in LS-BI may make food passage smoother, it may also induce gastritis, esophagitis and nutritional disorders due to bile reflux.

We, herein, compared postoperative endoscopic findings and nutritional status, as well as surgical outcomes, between LS-BI in TLDG and CS-BI in LADG. The aim of this study was to identify the advantages and disadvantages of TLDG, especially from the viewpoint of functional outcomes.

\section{Patients and Methods}

Patients. From December 2012 to March 2017, 140 patients underwent laparoscopic DG with BI for gastric cancer at our Institute. Of these, the present study targeted only patients who underwent LADG or TLDG with BI followed by upper endoscopy at 1 year after surgery. Patients who did not undergo endoscopy 1 year postoperatively, or in whom the endoscopic findings could not be determined with actual images were excluded. During this period, our principle indication for laparoscopic gastrectomy was early gastric cancer and cT2N0 gastric cancer according to the 
Japanese Classification of Gastric Cancer (7); therefore, patients with cT2N1 or more advanced gastric cancer were excluded from this study. Our first-choice reconstructive method after DG was BI, and Roux-en-Y gastrojejunostomy was employed when the size of the remnant stomach was small, gastric cancer invaded the duodenum, or evident hiatal hernia was co-existent. Our initial procedure was LADG with CS-BI, and TLDG with LS-BI was preferentially used from April 2014. All operations were performed or supervised by surgeons with adequate experience, and who were qualified by the Japan Society for Endoscopic Surgery. Ultimately, 100 ( 50 for LADG, and 50 for TLDG) patients were included in this retrospective study. This study was approved by the Institutional Review Board of our institute (ERB-C-1315).

Surgical procedures of $L A D G$ with $C S-B I$. The detailed surgical procedure of LADG with CS-BI was described in our previous report $(8,9)$. Briefly, after laparoscopic full lymph node dissection based on the Japanese Gastric Cancer Treatment Guidelines (10) and mobilization of the stomach, the distal stomach was pulled out through a $4 \mathrm{~cm}$ upper midline laparotomy. The anvil of a $29 \mathrm{~mm} \mathrm{CS}$ was inserted into the duodenal stump and a purse-string suture was tied over the anvil. The greater curvature side of the stomach was transected at an appropriate line using a $60 \mathrm{~mm}$ LS, leaving $5 \mathrm{~cm}$ of stomach wall uncut at the lesser curvature side. The CS was inserted through a small incision created at the uncut lesser curvature side of the stomach and the trocar of the CS was extended to penetrate the corner of the stapling line at the greater curvature. The trocar was connected to the anvil and gastroduodenostomy was performed by a hemi-double stapling technique. Finally, the gastrotomy at the lesser curvature of the stomach was closed with an LS, and the resected specimen was removed.

Surgical procedures of TLDG with LS-BI. LS-BI in TLDG was performed with the delta-shaped anastomosis firstly reported by Kanaya et al. (5). The duodenal bulb was transected using a $60 \mathrm{~mm}$ LS from the posterior to the anterior wall at the end of dissections of the suprapyloric and infrapyloric lymph nodes. After full lymph node dissection based on the Japanese Gastric Cancer Treatment Guidelines (10) and gastric mobilization, the stomach was transected using two or three $60 \mathrm{~mm}$ LSs and the resected specimen was removed through a minimally extended umbilical incision. Small incisions were created along the edge of the stomach and duodenum, and the posterior walls of both the stomach and the duodenum were approximated and joined with a $60 \mathrm{~mm}$ LS (suture length was from 40 to $50 \mathrm{~mm}$ ). After confirming there were no wall defects or bleeding, the common stab incision was closed using one or two applications of $60 \mathrm{~mm}$ LSs.

Surgical outcomes and postoperative complications. Surgical outcomes were obtained from our hospital records. Any complications occurring within 30 days after surgery or during a prolonged hospital stay were classified according to the ClavienDindo classification of surgical complications (11), and complications of grade II or more were reviewed.

Acute inflammatory response after surgery. The white blood cell (WBC) count and C-reactive protein (CRP) level at 1, 3 and 7 days after surgery were measured to compare postoperative acute inflammatory responses between LS-BI in TLDG and CS-BI in LADG.
Table I. Characteristics of patients with gastric cancer who underwent linear-stapled Billroth I (LS-BI) in totally laparoscopic distal gastrectomy and circular-stapled Billroth I (CS-BI) in laparoscopyassisted distal gastrectomy.

\begin{tabular}{lccc}
\hline Characteristic & $\begin{array}{c}\text { LS-BI } \\
(\mathrm{n}=50)\end{array}$ & $\begin{array}{c}\text { CS-BI } \\
(\mathrm{n}=50)\end{array}$ & $p$-Value \\
\hline Age, years & & & \\
$\quad$ Median (range) & $66(43-84)$ & $68(36-85)$ & 0.534 \\
Gender, $\mathrm{n}$ & & & \\
$\quad$ Male & 37 & 27 & 0.060 \\
Female & 13 & 23 & \\
BMI, kg/m & & & \\
Median (range) & $22.6(15.2-28.5)$ & $22.5(12.4-31.3)$ & 0.743 \\
Comorbidities, $\mathrm{n}(\%)$ & & & \\
Hypertension & $13(26.0)$ & $16(32.0)$ & 0.660 \\
Diabetes mellitus & $3(6.0)$ & $5(10.0)$ & 0.715 \\
Heart disease & $3(6.0)$ & $3(6.0)$ & $>0.99$ \\
Chronic liver disease & $3(6.0)$ & $1(2.0)$ & 0.617 \\
Chronic renal failure & $0(0)$ & $2(4.0)$ & 0.495 \\
Tumor location, $\mathrm{n}$ & & & \\
Middle third & 26 & 31 & 0.419 \\
lower third & 24 & 19 & \\
Clinical T-stage, $\mathrm{n}$ & & & \\
T1 & 44 & 43 & $>0.99$ \\
T2 & 6 & 7 & \\
Clinical N-stage, $\mathrm{n}$ & & & \\
N0 & 49 & 50 & \\
N1 & 1 & 0.99 \\
\hline
\end{tabular}

BMI: Body mass index.

Postoperative endoscopic findings and nutritional status. Endoscopic findings of the remnant stomach were evaluated according to the Residue, Gastritis, and Bile classification (12). The amount of residual food was classified into five grades: grade 0: no residual food; grade 1: a small amount of residual food; grade 2: a moderate amount of residual food, but possible to observe the entire surface of the remnant stomach with body rolling; grade 3: a moderate amount of residual food that hindered observation of the entire surface even with body rolling; and grade 4: a large amount of residual food, for which endoscopic observation was impossible. The degree of gastritis was defined in five categories: grade 0 , normal mucosa; grade 1: mild redness; grade 2: intermediate grade between grade 1 and grade 3 ; grade 3 : severe redness; and grade 4: apparent erosion. Bile reflux was defined as follows: grade 0: absence of bile reflux; and grade 1: presence of bile reflux. Endoscopic gastroesophageal reflux was evaluated according to the Los Angeles classification (13). The levels of serum total protein and albumin, and body weight at 6 months and 1 and 2 years after surgery were recorded to evaluate postoperative nutritional status.

Statistical analysis. All patients were divided into two groups according to the surgical procedure (LS-BI in TLDG and CS-BI in LADG). Differences between the groups were analyzed by Fisher's exact test for categorical variables and Student's $t$-test for continuous variables. All analyses were performed using JMP 
Table II. Surgical outcomes of patients with gastric cancer who underwent linear-stapled Billroth I (LS-BI) in totally laparoscopic distal gastrectomy and circular-stapled Billroth I (CS-BI) in laparoscopyassisted distal gastrectomy.

\begin{tabular}{|c|c|c|c|}
\hline Variable & $\begin{array}{l}\text { LS-BI } \\
(\mathrm{n}=50)\end{array}$ & $\begin{array}{l}\text { CS-BI } \\
(\mathrm{n}=50)\end{array}$ & $p$-Value \\
\hline \multicolumn{4}{|l|}{ Lymph node dissection, $n$} \\
\hline D1 & 42 & 44 & 0.774 \\
\hline $\mathrm{D} 1+/ \mathrm{D} 2$ & 8 & 6 & \\
\hline \multicolumn{4}{|l|}{ Combined resection, $\mathrm{n}(\%)$} \\
\hline Gall bladder & $3(6.0)$ & $3(6.0)$ & $>0.99$ \\
\hline \multicolumn{4}{|l|}{ Operative time, $\min$} \\
\hline Median (range) & $291(170-394)$ & $260(158-423)$ & 0.002 \\
\hline \multicolumn{4}{|l|}{ Blood loss, ml } \\
\hline Median (range) & $10(0-200)$ & $28(0-231)$ & 0.006 \\
\hline \multicolumn{4}{|l|}{ No. of lymph nodes retrieved } \\
\hline Median (range) & $39(12-63)$ & $39(13-71)$ & 0.591 \\
\hline \multicolumn{4}{|l|}{ Complication, $\mathrm{n}(\%)$} \\
\hline Total & $2(4.0)$ & $6(12.0)$ & 0.269 \\
\hline Anastomotic complication & $0(0)$ & $2(4.0)$ & 0.495 \\
\hline Leakage & $0(0)$ & $0(0)$ & - \\
\hline Stricture & $0(0)$ & $1(2.0)$ & $>0.99$ \\
\hline Bleeding & $0(0)$ & $1(2.0)$ & $>0.99$ \\
\hline Pancreatic fistula & $1(2.0)$ & $1(2.0)$ & $>0.99$ \\
\hline Intra-abdominal abscess & $1(2.0)$ & $0(0)$ & $>0.99$ \\
\hline Ileus & $0(0)$ & $1(2.0)$ & $>0.99$ \\
\hline Pneumonia & $0(0)$ & $2(4.0)$ & 0.495 \\
\hline Delayed gastric emptying & $0(0)$ & $0(0)$ & - \\
\hline Wound infection & $00)$ & $0(0)$ & - \\
\hline \multicolumn{4}{|l|}{ Mortality, n (\%) } \\
\hline Total & $0(0)$ & $0(0)$ & - \\
\hline \multicolumn{4}{|l|}{ Postoperative hospital stay, days } \\
\hline Median (range) & $10(5-20)$ & $12(8-69)$ & 0.069 \\
\hline
\end{tabular}

software (version 12; SAS Institute Inc., Cary, NC, USA), and values of $p<0.05$ were considered significant.

\section{Results}

Patient characteristics. Table I shows the characteristics of patients who underwent LS-BI in TLDG and CS-BI in LADG. Although the CS-BI group tended to include more male patients, no significant differences were found in terms of age, sex, body mass index, comorbidities, tumor location, or clinical $\mathrm{T}-$ and $\mathrm{N}$-stage between the two groups.

Surgical outcomes. Table II shows the surgical outcomes of patients who underwent LS-BI and CS-BI. No significant differences were observed in the extent of lymph node dissection, number of retrieved lymph nodes, and frequency of combined organ resection between the two groups. The operative time was longer $(p=0.002)$, and estimated blood loss was less $(p=0.006)$ in the LS-BI group. The overall
Table III. Endoscopic findings after linear-stapled Billroth I (LS-BI) in totally laparoscopic distal gastrectomy and circular-stapled Billroth I (CS-BI) in laparoscopy-assisted distal gastrectomy for gastric cancer.

\begin{tabular}{|c|c|c|c|}
\hline & $\begin{array}{l}\text { LS-BI } \\
(\mathrm{n}=50)\end{array}$ & $\begin{array}{l}\text { CS-BI } \\
(\mathrm{n}=50)\end{array}$ & $p$-Value \\
\hline \multicolumn{4}{|l|}{ Residual food grade } \\
\hline 0 & 40 & 32 & \\
\hline 1 & 4 & 7 & \\
\hline 2 & 5 & 8 & \\
\hline 3 & 1 & 3 & \\
\hline 4 & 0 & 0 & \\
\hline$\geq$ Grade $1, \mathrm{n}(\%)$ & $10(20.0)$ & $18(36.0)$ & 0.118 \\
\hline$\geq$ Grade $2, \mathrm{n}(\%)$ & $6(12.0)$ & $11(22.0)$ & 0.287 \\
\hline \multicolumn{4}{|l|}{ Gastritis grade } \\
\hline 0 & 13 & 20 & \\
\hline 1 & 14 & 21 & \\
\hline 2 & 14 & 7 & \\
\hline 3 & 9 & 2 & \\
\hline 4 & 0 & 0 & \\
\hline$\geq$ Grade $1, \mathrm{n}(\%)$ & $37(74.0)$ & $30(60.0)$ & 0.202 \\
\hline$\geq$ Grade $2, \mathrm{n}(\%)$ & $23(46.0)$ & $9(18.0)$ & 0.005 \\
\hline \multicolumn{4}{|l|}{ Bile reflux grade ${ }^{a}$} \\
\hline 0 & $38(76.0)$ & $40(80.0)$ & 0.810 \\
\hline 1 & $12(24.0)$ & $10(20.0)$ & \\
\hline \multicolumn{4}{|c|}{ Reflux esophagitis grade } \\
\hline A & 14 & 12 & \\
\hline B & 2 & 0 & \\
\hline $\mathrm{C}$ & 0 & 1 & \\
\hline $\mathrm{D}$ & 0 & 0 & \\
\hline$\geq$ Grade A, n (\%) & $16(32.0)$ & $13(26.0)$ & 0.660 \\
\hline
\end{tabular}

aAccording to the Residue, Gastritis, and Bile classification (12). bAccording to the Los Angeles classification (13).

complication rates were $4.0 \%$ in the LS-BI group and $12.0 \%$ in the CS-BI group $(p=0.269)$. There was no intraoperative anastomotic complication such as duodenal wall injury in either group. Although no anastomotic complications were observed postoperatively in the LS-BI group, the CS-BI group had two $(4.0 \%)$ anastomotic complications (one stricture and one bleeding) both of which required endoscopic treatment. The LS-BI group had one (2.0\%) pancreatic fistula and one (2.0\%) intra-abdominal abscess, while the CS-BI group had one (2.0\%) pancreatic fistula, one (2.0\%) paralytic ileus and two $(4.0 \%)$ pneumonia cases. No delayed gastric emptying or wound infection was found in either the LS-BI or CS-BI group, and no mortality was recorded in this series. The length of the postoperative hospital stay tended to be shorter in the LS-B-I group $(p=0.069)$.

Acute inflammatory response after surgery. Figure 1 shows the postoperative WBC count and CRP level in patients who underwent LS-BI or CS-BI. There were no significant 
A

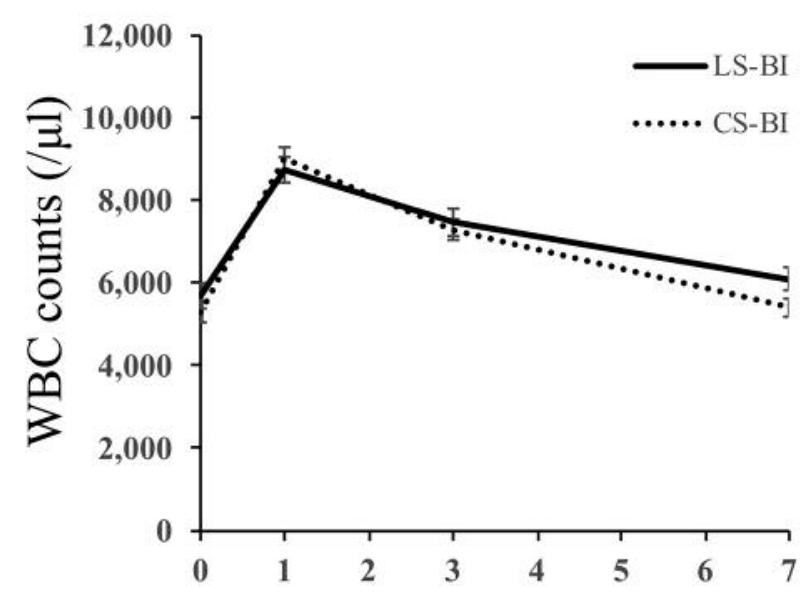

\section{Days after surgery}

B

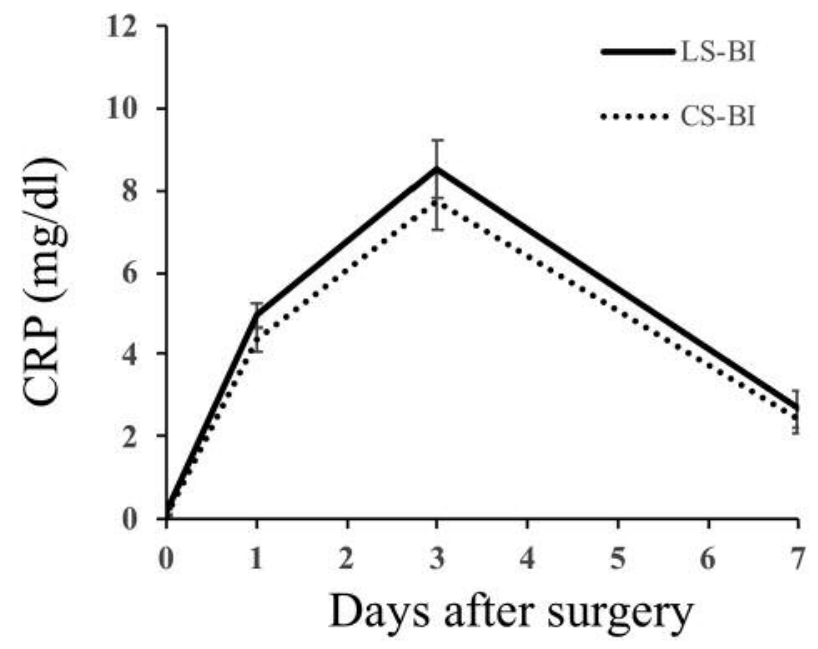

Figure 1. Comparison of postoperative acute inflammatory responses between patients with gastric cancer who underwent linear-stapled Billroth I (LS-BI) in totally laparoscopic distal gastrectomy and circularstapled Billroth I (CS-BI) in laparoscopy-assisted distal gastrectomy. A: white blood cell (WBC) counts. B: Plasma concentration of $C$-reactive protein (CRP). Data are presented as the mean $\pm S E$.

differences in these levels at 1, 3 and 7 days after surgery between the two groups, even though the WBC count at 7 days after surgery tended to be higher in the LS-BI group.

Postoperative functional outcomes. Table III shows the postoperative endoscopic findings in patients who underwent
A

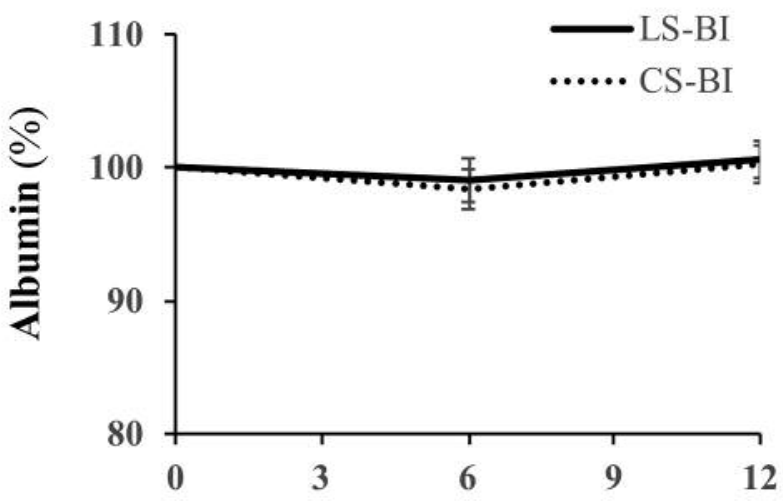

Months after surgery

B

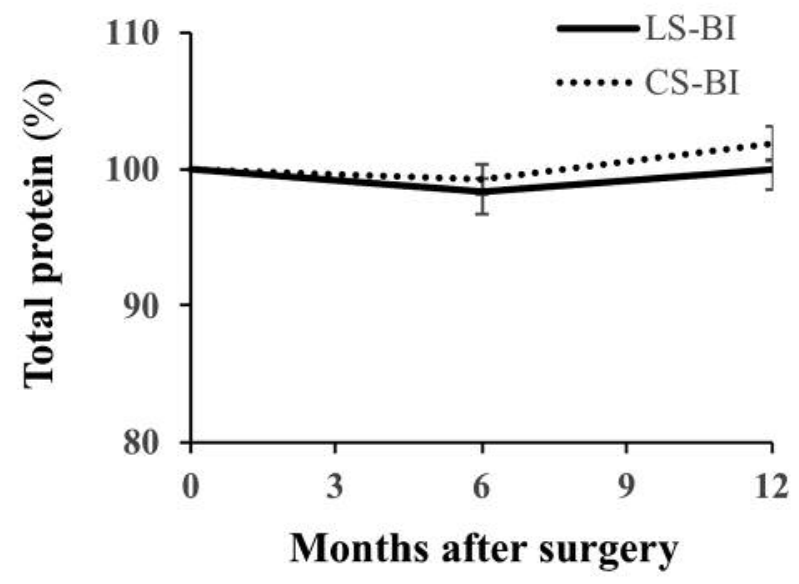

C

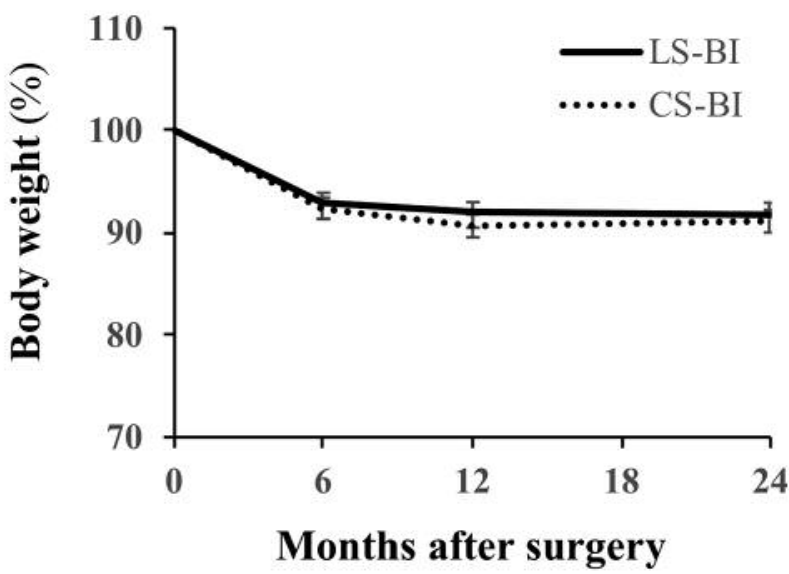

Figure 2. Comparisons of postoperative nutritional status between patients with gastric cancer who underwent linear-stapled Billroth I (LS-BI) in totally laparoscopic distal gastrectomy and circular-stapled Billroth I (CS-BI) in laparoscopy-assisted distal gastrectomy. A: Albumin. B: Total protein. C: Body weight. All postoperative data are presented as mean $\pm S E$ values relative to preoperative data. 
LS-BI or CS-BI. The incidence of residual food in the LS-B-I group (20.0\%) was lower than that in the CS-BI group (36.0\%), even though no significant difference was observed $(p=0.118)$. The occurrence of grade 2 or more severe gastritis in the LSB-I group (46.0\%) was significantly higher than that in the CSBI group $(18.0 \%)(p=0.005)$. As for bile reflux, there was no significant difference between the groups $(p=0.810)$. Reflux esophagitis was observed in $32 \%$ of the LS-BI group, and $26.0 \%$ of the CS-BI group ( $p=0.660$ ). The rates of proton pump inhibitor and camostat mesylate use at 1 year after surgery were $20.0 \%$ and $4.0 \%$ in the LS-BI group, and $10.0 \%$ and $2.0 \%$ in the CS-BI group ( $p=0.262$, and $>0.99$, respectively).

Figure 2 shows comparisons of postoperative nutritional status between LS-BI and CS-BI. The levels of serum total protein and albumin at 6 months and 1 year after surgery were not significantly different between the two groups. Furthermore, body weight relative to the preoperative measurement at 6 months and 1 and 2 years after surgery were $0.93,0.92$, and 0.92 in the LS-BI group, and 0.92, 0.91, and 0.91 in the CS-BI group and did not differ significantly $(p=0.646,0.438$ and 0.697 , respectively).

\section{Discussion}

TLDG certainly has a cosmetic benefit, and the omission of an upper midline incision can contribute to a reduction in postoperative pain, wound infection and intra-abdominal adhesion (14-16); however, it is debatable whether TLDG is really a less invasive procedure compared to LADG. Song et al. reported in a prospective study that there were no differences in the WBC count and CRP level or plasma cortisol concentrations at postoperative days 1 and 3 between TLDG and LADG (17). Similarly, the present study did not show any advantages of TLDG in terms of the postoperative WBC and CRP; therefore, the omission of upper midline laparotomy for reconstructions alone may not reduce surgical invasiveness, at least in laparoscopic DG.

Intracorporeal anastomosis may be technically difficult, especially for surgeons with little experience, while better visualization under a laparoscope can lead to safer anastomosis even in obese patients, for whom extracorporeal anastomosis is hard to perform with a limited working space. In the present study, although all operations were performed or supervised with surgeons qualified by the Japan Society for Endoscopic Surgery, the short-term outcomes of TLDG were not inferior to those of LADG, with the exception of the longer operative time. There was no intraoperative anastomotic complication such as duodenal wall injury in this series; therefore, LS-BI can be safely performed if surgeons are familiar with the intracorporeal use of LSs and laparoscopic suturing. The lower amount of blood loss in TLDG was probably attributable to the absence of an upper midline laparotomy and extracorporeal procedures.
Notably in this series, there were no anastomotic complications such as leakage, stricture or bleeding in the LS-BI group. In contrast, one anastomotic stricture and one case of anastomotic bleeding were observed in the CS-BI group. Okabe et al. reported that none of 184 patients undergoing LS-BI in TLDG experienced postoperative anastomotic stricture or bleeding (3); therefore, LS-BI may have some advantages in terms of reduced incidence of anastomotic stricture and bleeding because the size of the anastomosis is theoretically larger compared to CS-BI, and hemostasis can easily be confirmed from the luminal side through the entry hole during the surgery.

In the endoscopic evaluation, the occurrence of grade 2 or more severe remnant gastritis was significantly higher in the LS-BI group (46.0\%) than the CS-BI group (18.0\%). Lee et al. reported that the amount of food intake was larger in the LS-BI group due to the more straight-forward structural alignment between the remnant stomach and duodenum and wider anastomotic lumen, while the incidence of endoscopic bile reflux was significantly higher in the LS-BI group (70\%) compared to the CS-BI group (40\%) (18). Therefore, the higher incidence of bile reflux might be a factor contributing to the higher incidence of remnant gastritis after LS-BI, even though our study did not show any difference in bile reflux between the two groups. Because our study also showed no differences in the incidence of reflux esophagitis between the two groups, higher rates of proton pump inhibitor and camostat mesylate administration in the LS-BI group may have affected the incidence of endoscopic bile reflux and reflux esophagitis.

Comparisons of postoperative nutritional status between LS-BI and CS-BI were major points of interest in this study. Despite the higher incidence of remnant gastritis in the LSBI group, no significant differences were observed in the postoperative level of serum total protein, albumin or body weight between the two groups. Lee et al. also reported that postoperative nutritional indices (body weight change, albumin level, transferrin level, and total lymphocyte count) were almost the same under the two procedures, but only three parameters (albumin at 3 years, and total lymphocyte count at 1 and 3 years) were significantly higher in the LSBI group (18). Kanaya et al. reported a very low incidence $(1.3 \%)$ of dumping syndrome after LS-BI, despite the high incidence of endoscopic bile reflux (73.5\%) (19). Therefore, remnant gastritis due to bile reflux after LS-BI may not itself be a critical problem in terms of postoperative nutritional status in patients who do not have a poor oral ingestion or digestion absorption disorders.

This study included some limitations. There may be some biases because this was a retrospective study and the sample size was not large enough to identify real differences between the two groups. Another limitation was the lack of data on patients' subjective symptoms scored by validated questionnaires such as 
the Postgastrectomy Syndrome Assessment Scale (20); therefore, the associations between endoscopic findings and reflux symptoms remained unclear. Nevertheless, the present study showed a higher incidence of remnant gastritis and comparable nutritional status after LS-BI compared to CS-BI. The wider range of duodenal peeling in LS-BI may also be associated with the higher incidence of bile reflux, even though the Kocher maneuver was not performed for any patients in this series (21). The results of the present study need to be validated in prospective studies with larger sample sizes, and further discussions about the optimal size of anastomosis and range of duodenal mobilization are needed to further support the practice of TLDG with LS-BI.

\section{Conclusion}

TLDG with LS-BI appears to be a good alternative to LADG with CS-BI because of its excellent short-term outcomes and comparable nutritional outcomes, but with the disadvantage of higher remnant gastritis occurrence.

\section{Conflicts of Interest}

The Authors declare that they have no conflicts of interest in regard to this study.

\section{Authors' Contributions}

K. Katsurahara, and T. Kosuga helped in study concept and design. Acquisition, analysis, or interpretation of data was carried out by K. Katsurahara, T. Kosuga, and E. Otsuji. Statistical analysis was done by K. Katsurahara and T. Kosuga. Drafting the article was done by K. Katsurahara, T. Kosuga, and E. Otsuji. Critical revision of the article for important intellectual content was performed by all the Authors belonging to the surgery team of our Institute.

\section{References}

1 Katai H, Mizusawa J, Katayama H, Takagi M, Yoshikawa T, Fukagawa T, Terashima M, Misawa K, Teshima S, Koeda K, Nunobe S, Fukushima N, Yasuda T, Asao Y, Fujiwara Y and Sasako M: Short-term surgical outcomes from a phase III study of laparoscopy-assisted versus open distal gastrectomy with nodal dissection for clinical stage IA/IB gastric cancer: Japan Clinical Oncology Group Study JCOG0912. Gastric Cancer 20(4): 699-708, 2017. PMID: 27718137. DOI: 10.1007/s10120016-0646-9

2 Kitano S, Iso Y, Moriyama M and Sugimachi K: Laparoscopyassisted Billroth I gastrectomy. Surg Laparosc Endosc 4(2): 146148, 1994. PMID: 8180768.

3 Okabe H, Obama K, Tsunoda S, Tanaka E and Sakai Y: Advantage of completely laparoscopic gastrectomy with linear stapled reconstruction: A long-term follow-up study. Ann Surg 259(1): 109-116, 2014. PMID: 23549426. DOI: 10.1097/SLA. 0b013e31828dfa5d
4 Fukunaga T, Ishibashi Y, Oka S, Kanda S, Yube Y, Kohira Y, Matsuo $\mathrm{Y}$, Mori O, Mikami S, Enomoto $\mathrm{T}$ and Otsubo $\mathrm{T}$ : Augmented rectangle technique for Billroth I anastomosis in totally laparoscopic distal gastrectomy for gastric cancer. Surg Endosc, 2018. PMID: 29915985. DOI: 10.1007/s00464-0186266-1

5 Kanaya S, Gomi T, Momoi H, Tamaki N, Isobe H, Katayama T, Wada Y and Ohtoshi M: Delta-shaped anastomosis in totally laparoscopic Billroth I gastrectomy: New technique of intraabdominal gastroduodenostomy. J Am Coll Surg 195(2): 284-287, 2002. PMID: 12168979. DOI: $10.1016 / \mathrm{S} 1072-$ 7515(02)01239-5

6 Oki E, Tsuda Y, Saeki H, Ando K, Imamura Y, Nakashima Y, Ohgaki K, Morita M, Ikeda T and Maehara Y: Book-binding technique for Billroth I anastomosis during totally laparoscopic distal gastrectomy. J Am Coll Surg 219(6): e69-73, 2014. PMID: 25283741. DOI: 10.1016/j.jamcollsurg.2014.09.001

7 Japanese Classification of Gastric Carcinoma: 3rd English edition. Gastric Cancer 14(2): 101-112, 2011. PMID: 21573743. DOI: $10.1007 / \mathrm{s} 10120-011-0041-5$

8 Ichikawa D, Kubota T, Kikuchi S, Fujiwara H, Nakanishi M, Ikoma H, Okamoto K, Sakakura C, Ochiai T, Kokuba Y and Otsuji E: Intracorporeal Billroth-I anastomosis using a circular stapler by the abdominal wall lifting method in laparoscopyassisted distal gastrectomy. Surg Laparosc Endosc Percutan Tech 19(5): e163-166, 2009. PMID: 19851243. DOI: 10.1097/ SLE.0b013e3181b6c 867

9 Kosuga T, Ichikawa D, Komatsu S, Kubota T, Okamoto K, Konishi H, Shiozaki A, Fujiwara $\mathrm{H}$ and Otsuji E: Clinical and surgical factors associated with organ/space surgical site infection after laparoscopic gastrectomy for gastric cancer. Surg Endosc 31(4): 1667-1674, 2017. PMID: 27506433. DOI: 10.1007/s00464-016-5156-7

10 Japanese Gastric Cancer Treatment Guidelines 2014 (ver. 4). Gastric Cancer 20(1): 1-19, 2017. PMID: 27342689. DOI: 10.1007/s10120-016-0622-4

11 Dindo D, Demartines N and Clavien PA: Classification of surgical complications: A new proposal with evaluation in a cohort of 6336 patients and results of a survey. Ann Surg 240(2): 205-213, 2004. PMID: 1360123. DOI: 00000658-20040800000003

12 Kubo M, Sasako M, Gotoda T, Ono H, Fujishiro M, Saito D, Sano $\mathrm{T}$ and Katai H: Endoscopic evaluation of the remnant stomach after gastrectomy: Proposal for a new classification. Gastric Cancer 5(2): 83-89, 2002. PMID: 12111583. DOI: $10.1007 / \mathrm{s} 101200200014$

13 Armstrong D, Bennett JR, Blum AL, Dent J, De Dombal FT, Galmiche JP, Lundell L, Margulies M, Richter JE, Spechler SJ, Tytgat GN and Wallin L: The endoscopic assessment of esophagitis: A progress report on observer agreement. Gastroenterology 111(1): 85-92, 1996. PMID: 8698230. DOI: 10.1053/gast.1996.v111.pm8698230

14 Ding W, Tan Y, Xue W, Wang Y and Xu XZ: Comparison of the short-term outcomes between delta-shaped anastomosis and conventional Billroth I anastomosis after laparoscopic distal gastrectomy: A meta-analysis. Medicine 97(9): e0063, 2018. PMID: 5851755. DOI: 10.1097/MD.0000000000010063

$15 \mathrm{Hu}$ GY, Tao F, Ji KW and Wang W: Comparison of delta-shape anastomosis and extracorporeal Billroth I anastomosis after laparoscopic distal gastrectomy for gastric cancer: A systematic 
review with meta-analysis of short-term outcomes. PLoS One 11(9): e0162720, 2016. PMID: 5025198. DOI: 10.1371/ journal.pone. 0162720

16 Wang SY, Hong J and Hao HK: A comparative study of deltashaped and conventional Billroth I anastomosis after laparoscopic distal gastrectomy for gastric cancer. Surg Endosc 31(8): 3191-3202, 2017. PMID: 27864720. DOI: 10.1007/ s00464-016-5344-5

17 Song KY, Park CH, Kang HC, Kim JJ, Park SM, Jun KH, Chin $\mathrm{HM}$ and Hur H: Is totally laparoscopic gastrectomy less invasive than laparoscopy-assisted gastrectomy?: Prospective, multicenter study. J Gastrointest Surg 12(6): 1015-1021, 2008. PMID: 18256884. DOI: $10.1007 / \mathrm{s} 11605-008-0484-0$

18 Lee HH, Song KY, Lee JS, Park SM and Kim JJ: Delta-shaped anastomosis, a good substitute for conventional Billroth I technique with comparable long-term functional outcome in totally laparoscopic distal gastrectomy. Surg Endosc 29(9): 2545-2552, 2015. PMID: 25427413. DOI: 10.1007/s00464-0143966-Z

19 Kanaya S, Kawamura Y, Kawada H, Iwasaki H, Gomi T, Satoh $\mathrm{S}$ and Uyama I: The delta-shaped anastomosis in laparoscopic distal gastrectomy: Analysis of the initial 100 consecutive procedures of intracorporeal gastroduodenostomy. Gastric Cancer 14(4): 365-371, 2011. PMID: 21573920. DOI: 10.1007/ s10120-011-0054-0
20 Nakada K, Ikeda M, Takahashi M, Kinami S, Yoshida M, Uenosono Y, Kawashima Y, Oshio A, Suzukamo Y, Terashima $\mathrm{M}$ and Kodera $\mathrm{Y}$ : Characteristics and clinical relevance of Postgastrectomy Syndrome Assessment Scale (PGSAS)-45: Newly developed integrated questionnaires for assessment of living status and quality of life in postgastrectomy patients. Gastric Cancer 18(1): 147-158, 2015. PMID: 24515247. DOI: 10.1007/s10120-014-0344-4

21 Misawa K, Terashima M, Uenosono Y, Ota S, Hata H, Noro H, Yamaguchi K, Yajima H, Nitta T and Nakada K: Evaluation of postgastrectomy symptoms after distal gastrectomy with Billroth-I reconstruction using the Postgastrectomy Syndrome Assessment Scale-45 (PGSAS-45). Gastric Cancer 18(3): 675681, 2015. PMID: 25091080. DOI: 10.1007/s10120-014-0407-6

Received July 11, 2019

Revised July 19, 2019

Accepted July 25, 2019 